\title{
Clinical features, management and in-hospital outcome of ST elevation myocardial infarction (STEMI) in young adults under $\mathbf{4 0}$ years of age
}

\author{
Aspetti clinici, trattamento e prognosi ospedaliera \\ di giovani adulti con STEMI e meno di 40 anni
}

\author{
Seyed Kianoosh Hosseini1, Abbas Soleimani1, Abbas Ali Karimi2, Saeed Sadeghian1, \\ Sirous Darabian1, Seyed Hesameddin Abbasi3 ${ }^{3}$ Seyed Hossein Ahmadi2, \\ Arezou Zoroufian1, Mehran Mahmoodian3, Ali Abbasi1,4
}

\begin{abstract}
Clinical features, management and in-hospital outcome of ST elevation myocardial infarction (STEMI) in young adults under 40 years of age. S.K. Hosseini, A. Soleimani, A.A. Karimi, S. Sadeghian, S. Darabian, S.H. Abbasi, S.H. Ahmadi, A. Zoroufian, M. Mahmoodian, A. Abbasi.

Objectives: This study was designed to evaluate the demographic and clinical findings and in-hospital management and outcome in patients with an acute ST-segment elevation myocardial infarction (STEMI).

Material and methods: By review of the Cardiovascular Tehran Heart Center Registry (CVDTHCR), 2028 patients were found to have the acute STEMI. We compared the patients' characteristics in $109(5.4 \%)$ subjects $\leq 40$ and 1919 subjects $>\mathbf{4 0}$ years old.

Results: The young patients had less diabetes, hypertension, dyslipidemia and history of MI or prior revascularization, and were more likely to be male $(92.7 \%$ vs. $74 \%)$,
\end{abstract}

smoker $(58.7 \%$ vs. $31.7 \%)$ and have family history of CVD $(50.5 \%$ vs. $23.4 \%)$. The young patients had higher prevalence of angiographically normal coronary artery $(13.7 \%$ vs. $0.9 \% ; \mathbf{p}<0.001)$. The young patients were more likely to undergo percutaneous coronary intervention $(38.5 \%$ vs. $18.6 \%$ ), whereas coronary artery bypass grafting was more common in the old ones $(p<0.001)$. In-hospital death was markedly different among young and old patients $(0.9 \%$ and $6.1 \%$, respectively; $p<0.01$ ).

Conclusion: In STEMI population, the risk profile, clinical findings and severity of coronary disease of the young differ substantially from the elderly counterparts. Young patients with STEMI have a favorable outcome compared with that in older patients.

Keywords: ST-segment elevation, myocardial infarction, risk factors, young, coronary artery disease.

Monaldi Arch Chest Dis 2009; 72: 71-76.

\footnotetext{
1 Department of Cardiology, Tehran Heart Center, Tehran University of Medical Sciences, Tehran, Iran.

2 Department of Cardiac Surgery, Tehran Heart Center, Tehran University of Medical Sciences, Tehran, Iran.

3 Department of Research, Tehran Heart Center, Tehran University of Medical Sciences, Tehran, Iran.

4 Department of Epidemiology, University Medical Center Groningen, Groningen, the Netherlands.
}

Corresponding author: Kianoosh Hosseini; North Kargar Street, Tehran Heart Center, Postal code 1411713138, Tehran, Iran; Tehran Heart Center, Tehran University of Medical Sciences; Tel. and fax No.: +98 2188029256 ; E-mail address: skhoseini@yahoo.com,aliiabbasi@yahoo.com

\section{Introduction}

Over the past three decades, mortality and morbidity attributable to cardiovascular diseases (CVD) have declined in several developed countries, whereas they appear to have increased substantially in many developing countries $[1,2]$. ST-segment elevation myocardial infarction (STEMI) is one of the common causes of cardiac consultation and admission at emergency departments and currently accounts for the high burden on health care services. The mortality, incidence, and clinical presentation of CVD tend to vary greatly over the time. During recent years, the mean age of CVD has decreased, but its prevalence has increased in Iran. Also, several studies have shown significant differences in the risk factors profile and prognosis of MI between different age groups [3-7].
There is a paucity of data in the existing literature on young Iranian patients with STEMI. In an attempt to characterize the acute STEMI patients $\leq 40$ years of age and older at our institution, we reviewed the Tehran Heart Center Cardiovascular Disease Registry (THCCVDR) and compared the demographics, clinical findings, and in-hospital outcome of these two groups of patients.

\section{Methods}

A total of 14848 patients who were admitted to our institution and had identification data in the THCCVDR between July 2003 and August 2007 were reviewed. Our study population was consisted of 2028 patients with STEMI from the THCCVDR. The THCCVDR contains patient data collected by 
cardiologists and trained general practitioners. The validity of all the data is checked by reabstracting $10 \%$ of the patients' entries and by reentering $5 \%$ of the patients' records. The study protocol was approved by the institutional Review Board, overseeing the participation of human subjects in research at Tehran University of Medical Sciences. This study conforms to the principles outlined in the Declaration of Helsinki.

The validation of AMI was based on information on medical history, symptoms, electrocardiograms, and cardiac enzymes. STEMI was diagnosed by a cardiologist when new or presumed new ST-segment elevation $\geq 1 \mathrm{~mm}(\geq 2 \mathrm{~mm}$ in V1 to V3) was seen in any location in two or more contiguous leads or new left bundle branch block was found on the index or qualifying electrocardiograms with $\geq 1$ positive cardiac biochemical marker of necrosis, including CK$\mathrm{MB}$ or cardiac troponin-I measurements. To characterize the young patients, 2028 patients with STEMI were classified into two groups of 109 subjects $\leq 40$ (young patients) and 1919 subjects > 40 years old. The following data were included for analysis: demographic data, namely age and gender, and CVD risk factors profile, comprised of current cigarette smoking history (patient regularly smokes a tobacco product/products one or more times per day or has smoked in the 30 days prior to admission), dyslipidemia (total cholesterol $\geq 5.0$, HDL-cholesterol $\leq 1.0$ in men or $\leq 1.1$ in women, triglycerides $\geq 2.0$ $\mathrm{mmol} / \mathrm{l}$ ), family history of CVD (first-degree relatives before the age of 55 in men and 65 years in women), hypertension (systolic blood pressure $\geq 140$ and/or diastolic $\geq 90 \mathrm{mmHg}$ and/or on anti-hypertensive treatment), diabetes mellitus (symptoms of diabetes and plasma glucose concentration $\geq 200 \mathrm{mg} / \mathrm{dl}$
(11.1 mmol/l), or fasting plasma glucose $\geq 126 \mathrm{mg} / \mathrm{dl}$ (7.0 mmol/l) or $2-\mathrm{hp} \geq 200 \mathrm{mg} / \mathrm{dl}(11.1 \mathrm{mmol} / \mathrm{l}))$, and opium consumption [8]. The cardiac status of the patients, namely acute coronary symptoms, clinical manifestations, MI location based on electrocardiographic findings, cardiac enzyme profile, left ventricular ejection fraction (LVEF), angiographic findings, and management and in-hospital outcomes were reported. In the patients who underwent a coronary angiography at the time of hospitalization, significant coronary artery disease (CAD) was defined as a diameter stenosis $>50 \%$ in each major epicardial artery. A narrowing of $<50 \%$ was considered mild CAD. Normal vessels were defined as the complete absence of any disease, including lack of even mild luminal irregularities, which were considered as evidence of early atherosclerosis.

The results were reported as mean \pm standard deviation $(\mathrm{SD})$ or median with $1^{\text {st }}$ and 3 rd quartiles (whenever the data did not appear to have normal distribution) for the quantitative variables, and the categorized variables were summarized as frequencies and percentages. The groups were compared using the Student's t-test or Mann-Whitney U for the continuous variables, and the $\chi 2$ test for the dichotomous variables. This study was done with the power of $90 \%$. P values of 0.05 or less were considered statistically significant. All the statistical analyses were carried out via Statistical Package for Social Sciences version 16 (SPSS Inc, Chicago, Illinois, USA).

\section{Results}

The demographic and historical characteristics of the study population are listed in Table 1 . The mean patient age was $60.6 \pm 12.5$ [20-112] years, and

Table 1. - Demographic and historical characteristics of patients with STEMI $(n=2028)$

\begin{tabular}{|c|c|c|c|c|}
\hline & Pts $\leq 40$ years $(n=109)$ & Pts $>40$ years $(n=1919)$ & All pts $(n=2028)$ & p Value \\
\hline Age (years) & $35.6 \pm 4.9$ & $62.0 \pm 11.3$ & $60.6 \pm 12.5$ & $<0.001$ \\
\hline Male gender & $101(92.7)$ & $1421(74)$ & $1522(75)$ & $<0.001$ \\
\hline \multicolumn{5}{|l|}{ Marital Status } \\
\hline Married & $86(78.9)$ & $1558(81.2)$ & $1644(81.1)$ & \\
\hline Single & $12(11)$ & $15(0.8)$ & $27(1.3)$ & \\
\hline Divorced & $1(0.9)$ & $10(0.5)$ & $11(0.5)$ & \\
\hline Widowed & 0 & $183(9.5)$ & $183(9.0)$ & $<0.001$ \\
\hline Family history of CVD & $55(50.5)$ & $450(23.4)$ & $505(24.9)$ & $<0.001$ \\
\hline Hypertension & $20(18.3)$ & $848(44.2)$ & $868(42.8)$ & $<0.001$ \\
\hline Diabetes mellitus & $11(10.1)$ & $645(33.6)$ & $656(32.3)$ & $<0.001$ \\
\hline Active cigarette Smoking & $64(58.7)$ & $609(31.7)$ & $673(33.2)$ & $<0.001$ \\
\hline Dyslipidemia & $36(33)$ & $911(47.5)$ & 947 (46.7) & 0.003 \\
\hline BMI & $26.6 \pm 4.2$ & $26.5 \pm 4.3$ & $26.5 \pm 4.3$ & 0.759 \\
\hline Opium use & $16(14.7)$ & $197(10.3)$ & $213(10.5)$ & 0.144 \\
\hline Prior MI & $2(1.8)$ & $90(4.7)$ & $92(4.5)$ & 0.504 \\
\hline Prior catheterization & $9(8.3)$ & $284(14.8)$ & $293(14.4)$ & 0.135 \\
\hline Normal prior angiogram & $2(1.8)$ & $4(0.2)$ & $6(0.3)$ & 0.133 \\
\hline Prior PCI & $3(2.8)$ & $62(3.2)$ & $65(3.2)$ & 0.375 \\
\hline Prior CABG & 0 & $48(2.5)$ & $48(2.5)$ & 0.154 \\
\hline Prior heart failure & 0 & $12(0.6)$ & $12(0.6)$ & 0.258 \\
\hline Prior CVA & 0 & $71(3.7)$ & $71(3.5)$ & 0.053 \\
\hline
\end{tabular}


$75 \%$ of the patients were male. Risk factors included smoking in $33.2 \%$ of the cases, dyslipidemia in $46.7 \%$, diabetes in $32.3 \%$, and hypertension in $42.8 \%$ with an average body mass index (BMI) of $26.5 \pm 4.3$ in this population. Family history of CVD was reported in $24.9 \%$. Use of opium as a novel CVD risk factor [8] was found in $10.5 \%$.

The frequency of the atherosclerotic risk factors and demographic and clinical characteristics for the patients $\leq 40$ and $>40$ years old was compared. Among the young patients, the frequency of the males, family history of CVD, and smoking was significantly more prevalent than that of the others $(\mathrm{P}<0.001)$. The old patients were more likely to have diabetes mellitus $(\mathrm{P}<0.001)$, hypertension $(\mathrm{P}<0.001)$, and dyslipidemia $(\mathrm{P}=0.003)$. In contrast, there was no significant statistical difference in BMI and opium use between the two groups.

The acute coronary symptoms, the chief complaints, Killiip Class, and MI locations were similar between the young and old patients. In echocardiography, the young patients had a significantly higher
LVEF in comparison with the old ones $(48.6 \pm 10.6$ vs. $42.5 \pm 12.6 ; \mathrm{P}<0.001)$. The arrhythmias were found more prevalent in the old patients; they were not, however, significantly different from the young patients. The serum level of the cardiac biochemical markers of necrosis was higher in the young patients (Table 2).

Coronary angiography was performed for 960 (43.3\%) patients; the young patients were more likely to undergo a coronary angiography (67\% vs. $46.2 \%$; P $<0.001)$. Significant coronary artery lesions were found in 688 patients $(74.8 \%)$, mostly presented as multi-vessel disease $(71.7 \%)$. The prevalence of significant left main coronary artery (LMCA) disease was low among all the patients (2.3\%). The significant coronary lesions mainly affected the left anterior descending (LAD) coronary artery ( 823 patients, $87.1 \%$ ). There was a statistically higher prevalence in coronary atherosclerotic vessels, for the multi-vessel, LAD, right coronary artery (RCA), and left circumflex artery (LCx) in the old patients. The young patients had a higher prevalence

Table 2. - Clinical, laboratory, and angiographic characteristics of patients with STEMI $(n=2028)$

\begin{tabular}{|c|c|c|c|c|}
\hline & Pts $\leq 40$ years $(n=109)$ & Pts $>40$ years $(n=1919)$ & All pts $(n=2028)$ & p Value \\
\hline Chest discomfort & $106(97.2)$ & $1831(95.4)$ & $1937(95.7)$ & 0.483 \\
\hline Typical chest pain & $77(70.6)$ & $1407(73.3)$ & $1484(73.2)$ & \\
\hline Atypical chest pain & $27(24.8)$ & $369(19.2)$ & $396(19.5)$ & \\
\hline Without chest pain & $5(4.6)$ & $143(7.5)$ & $148(7.2)$ & 0.244 \\
\hline \multicolumn{5}{|l|}{ Pain duration } \\
\hline$\leq 30 \mathrm{~min}$ & $36(33)$ & $716(37.3)$ & $752(37.1)$ & \\
\hline$>30 \mathrm{~min}$ & $73(67)$ & $1203(62.7)$ & $1276(92.9)$ & 0.540 \\
\hline Dyspnea & 17 (15.6) & $357(18.6)$ & $374(18.5)$ & 0.425 \\
\hline Palpitation & $5(4.6)$ & $60(3.1)$ & $65(3.2)$ & 0.402 \\
\hline Chest pain still present & $70(64.3)$ & $1112(57.9)$ & $1937(95.7)$ & 0.185 \\
\hline \multicolumn{5}{|l|}{ Killip class } \\
\hline I & $55(93.2)$ & $916(82)$ & $971(82.6)$ & \\
\hline II & $4(6.8)$ & $148(13.2)$ & $152(12.9)$ & \\
\hline III & 0 & $23(2.1)$ & $23(2)$ & \\
\hline IV & 0 & $30(2.7)$ & $30(2.6)$ & 0.224 \\
\hline CK-MB level (IU/L) & $104(55,180.7)$ & $78.5(38,161)$ & $80(39,161.2)$ & 0.019 \\
\hline Troponin I level $(\mu \mathrm{g} / \mathrm{L})$ & $8.2(2.4,24,7)$ & $5.1(1.6,16)$ & $5.2(1.7,16.5)$ & 0.031 \\
\hline LVEF in echocardiography & $48.6 \pm 10.6$ & $42.5 \pm 12.6$ & $42.8 \pm 12.5$ & $<0.001$ \\
\hline Anterior MI & $33(30.3)$ & $544(28.3)$ & $577(28.5)$ & 0.836 \\
\hline Inferior MI & $49(45)$ & $697(36.3)$ & $746(36.8)$ & 0.252 \\
\hline Lateral MI & $8(7.3)$ & $83(4.3)$ & $91(4.5)$ & 0.414 \\
\hline Anteroseptal MI & $10(9.2)$ & $289(15.1)$ & $299(14.7)$ & 0.331 \\
\hline Angiography & $73(67)$ & $887(46.2)$ & $960(47.3)$ & $<0.001$ \\
\hline Normal coronary angiogram & $11(13.7)$ & $8(0.9)$ & $18(1.9)$ & $<0.001$ \\
\hline Mild coronary disease & $1(1.4)$ & $20(2.3)$ & $21(2.2)$ & \\
\hline Single-vessel disease & $29(39.7)$ & $204(33)$ & $233(24.3)$ & \\
\hline Multi- vessel disease & $33(45.2)$ & $655(73.8)$ & $519(54.1)$ & $<0.001$ \\
\hline \multicolumn{5}{|l|}{ LMCA disease } \\
\hline$\leq 50 \%$ & $1(1.4)$ & $59(6.7)$ & $60(6.2)$ & \\
\hline$>50 \%$ & 0 & $23(2.6)$ & $23(2.4)$ & 0.073 \\
\hline LAD disease & $50(68.5)$ & $773(87.1)$ & $823(85.7)$ & $<0.001$ \\
\hline LCx disease & $26(35.6)$ & $538(60.7)$ & $564(56.8)$ & $<0.001$ \\
\hline RCA disease & $36(49.3)$ & $588(66.3)$ & $624(65)$ & 0.003 \\
\hline
\end{tabular}

CVD, cardiovascular disease; PCI, percutaneous coronary intervention; CABG, coronary artery bypass grafting; CVA, cerebrovascular accident; MI, myocardial infarction; LMCA, left main coronary artery; LAD, left anterior descending; LCx, left circumflex; RCA, right coronary artery 
of angiographically normal coronary artery $(13.7 \%$ vs. $0.9 \% ; \mathrm{P}<0.001$ ) (Table 2 ).

Percutaneous coronary intervention (PCI) and coronary artery bypass grafting $(\mathrm{CABG})$ of the infarct-related lesions were performed in 398 (19.6\%) and $433(21.4 \%)$ patients, respectively. There was an age-related difference for revascularization options. The young patients were more likely to undergo PCI (38.5\% vs. 18.6\%; $\mathrm{P}<0.001)$, whereas CABG was more common in the old ones $(22.1 \%$ vs. $18.6 \%$; $\mathrm{P}<0.001)$. The others were put on a medical treatment. The length of stay in hospital was significantly shorter for the young patients $(\mathrm{p}<0.001)$. There was no cerebrovascular accident or recurrent major adverse coronary events in the young patients. In-hospital mortality occurred more frequently in the elderly patients compared with the young ones $(6.1 \%$ vs. $0.9 ; \mathrm{P}<0.01)$ (Table 3$)$.

\section{Discussion}

The THCCVDR has been established as a prospective registry that describes the epidemiology, management practices, and in-hospital outcome of patients with the entire spectrum of ACSs or suspected to have an ACS. The proportion of acute STEMI amounted to $13.6 \%$ of all the Registry population during the four-year period. In real-life, there are substantial differences between patient populations and clinical trial patient populations. In addition, there is considerable heterogeneity in patient management practices [9-11]. Therefore, the large-scale observational data sets are important to complement the information obtained via randomized clinical trials. The current study showed a significantly different clinical, angiographic, and biochemical profile in younger patients with coronary artery disease undergoing coronary angiography compared with older patients. However, it should be noted that these findings were based on the epidemiology of CVD, health care facilities and services in our region.
The STEMI in young adults may have different atherosclerotic risk factors profile. Our results revealed that the male gender, smoking, and family history of CVD were more common in the younger patients. The present data are consistent with an overwhelming body of evidence that the atherosclerotic process starts in adolescence or early adulthood, insidiously progressing under the influence of various risk factors. As in the present study, in which about $60 \%$ of the patients were smokers, prior observations documented active tobacco abuse in 65-92\% of young patients with MI [6, 12-26]. Concordantly, almost all the previous studies have mentioned that male patients and those with a family history of CVD have the propensity to earlier ACSs [19-23].

As expected, there was a lower incidence of hypertension, dyslipidemia, and diabetes in our younger patients, which is in agreement with previous studies $[12-14,20,21,27]$. It is related to the long-term role of these metabolic and endocrine disorders in the atherosclerosis process and ACSs, whereas cigarette smoking may be associated with thrombogenicity and spasm of the coronary arteries [28, 29]. In our young patients, interactions between gender, family history, and smoking and metabolic syndrome [30] were tested, but no significant effect modification was found. Prescott et al. [31] also found no interaction between smoking and other MI risk factors on MI risk. Most of our young patients were male, smokers, or who had a family history of CVD. These findings suggest that coronary disease may have different predisposing conditions in this population. It seems to be concordant with the predominant role of men in the socioeconomic activities of Iranian families in comparison with women. The difficult and time-consuming financial commitments of males (e.g., males who work two or more shifts) can be assumed to be the culprit for an earlier development of CVD earlier. Further investigation needs to evaluate the effect of socioeconomic status and psychological stress of the society on young patients. Early lifestyle modifications and

Table 3. - Treatment and outcome of patients with STEMI $(n=2028)$

\begin{tabular}{lllll}
\hline & Pts $\leq \mathbf{4 0}$ years $(\mathbf{n = 1 0 9})$ & Pts $>$ 40 years $(\mathbf{n = 1 9 0 9})$ & All pts $(\mathbf{n = 2 0 2 8})$ & $\mathbf{p ~ V a l u e ~}$ \\
\hline LOS in hospital (days) & $6.2 \pm 3.4(1-17)$ & $8.1 \pm 5(1-37)$ & $8 \pm 4.9(1-37)$ & 0.031 \\
Atrial fibrillation & 0 & $33(1.7)$ & $33(1.6)$ & 0.257 \\
Ventricular tachycardia & 0 & $5(0.3)$ & $5(0.2)$ & 0.486 \\
Ventricular fibrillation & 0 & $15(0.8)$ & $15(0.7)$ & 0.435 \\
RBBB & $1(0.9)$ & $31(1.6)$ & $32(1.6)$ & 0.724 \\
LBBB & $1(0.9)$ & $38(2.0)$ & $39(1.9)$ & 0.720 \\
AV block & 0 & $27(1.4)$ & $27(1.3)$ & 0.397 \\
PVC & $2(1.8)$ & $24(1.3)$ & $26(1.3)$ & 0.647 \\
Acute MR & $5(4.6)$ & $143(7.5)$ & $148(7.3)$ & 0.519 \\
CVA & 0 & $6(0.3)$ & $6(0.3)$ & 0.053 \\
Heart failure & $2(1.8)$ & $109(5.7)$ & $111(5.5)$ & 0.258 \\
Ventricular aneurysm & 0 & $11(0.6)$ & $11(0.5)$ & 0.677 \\
PCI & $42(38.5)$ & $356(18.6)$ & $398(19.6)$ & $<0.001$ \\
CABG & $9(8.3)$ & $424(22.1)$ & $433(21.4)$ & $<0.001$ \\
Death & $1(0.9)$ & $117(6.1)$ & $118(5.8)$ & 0.010 \\
\hline
\end{tabular}

LOS, length of stay; RBBB, right bundle branch block; LBBB, left bundle branch block, AV, atrioventricular; PVC, premature ventricular contraction; MR, mitral replacement; CVA, cerebrovascular accident; PCI, percutaneous coronary intervention; CABG, coronary artery bypass grafting 
pharmacological interventions should take into account smoking, dyslipidemia, and body weight control, particularly in men. It should be noted that hypertension and diabetes were less frequent at the age of our cohort. A general notion has evolved that the intensity of preventive efforts should be adjusted to a patient's risk for developing CVD, i.e., the higher the risk, the more aggressive the intervention should be.

Our findings that a normal coronary angiogram was more frequent in the young patients and that the young patients had a higher frequency of single-vessel disease as compared to the old patients are consistent with previous reports [6, 12, 13, 32, 33]. There is an overwhelming body of evidence that the atherosclerotic process starts in adolescence or early adulthood, insidiously progressing under the influence of various risk factors. Autopsy observations have shown that the occurrence of MI in young people with cardiovascular risk factors could be the expression of a premature and severe atherosclerotic process [34].

Not surprisingly, we observed that the rate of adverse outcomes (i.e., dysrhythmia, cerebrovascular accident, heart failure, and death) was lower in the younger patients, although we could not determine the relative contribution of younger age versus the more aggressive diagnostic procedures that younger patients received. The young patients in our study were more likely to have PCI as their coronary revascularization, whereas $\mathrm{CABG}$ was the more common revascularization option in the elderly patients. Importantly, the relative proportion of in-hospital mortality was significantly lower in the young age group $(0.9 \%$ vs. $6.1 \%)$. In contrast to their younger counterparts, the elderly patients often presented with a more complex cardiovascular risk profile and prior events. Our young patients were less likely to have had a history of MI and prior revascularization, whereas the old patients had lower LVEF in comparison with the younger ones. In the present study, the rate of overall in-hospital mortality was $5.8 \%$, which is comparable with the $15.1 \%$ mortality rate from the academic center in Tehran, Iran [3]. The previous reports from developed countries were $7 \%$ for STEMI and 6\% for non-STEMI patients in the Global Registry of Acute Coronary Events (GRACE) Registry [35] and 7.4\% in a study in the USA by Gheorghiade et al. [36]. In the The Polish Registry of Acute Coronary Syndromes, the nonTEMI patients had lower mortality (6.6 vs. $9.3 \%, \mathrm{p}$ $<0.0001)$ compared with the STEMI patients [37]. In the PRIAMHO II study, the outcome was better in the younger group, with a lower mortality rate at 28 days $(3.7 \%)$, demonstrating that age $<45$ years was an independent protective factor for mortality of AMI [38]. In a very recent study by Anderson et al, the VALIANT trial [39], outcomes of the young patients with AMI was substantively better than their older counterparts.

The current analysis is strengthened by the diversity and size of the population studied; be that as it may, a number of limitations should be noted. Although patients $\leq 40$ years of age comprised just $5.4 \%$ of the total STEMI population in the THCCVDR, this analysis of 2028 patients of STEMI represents one of the largest studies to focus on patients after infarction. As this study population represented a relatively high-risk cohort of patients with MI, we remain cautious in generalizing these results to a broader group of lower risk, post-MI patients. Furthermore, our patients had been followed prospectively through other registries (i.e., Angiography, Angioplasty, and $\mathrm{CABG}$ ); nevertheless, the present study, being a single-center survey may have lost some data of the patients who were not admitted. In other words, the majority of findings were related to local epidemiology of CVD, management habits, and facilities of our center. Some long-term follow-up events, particularly death and stroke, were not verified by checking the subjects' hospital records; and in these circumstances, the patients' declarations (or statements of their relatives) were accepted as true. Accordingly, the present analysis cannot claim to represent the findings for all patients early after MI.

In conclusion, we found that cigarette smoking, male gender, and a family history of cardiovascular diseases were more prevalent in young patients with STEMI, whereas the elderly patients were more likely to have dyslipidemia, hypertension, and diabetes. Although the young patients had less complex clinical profiles and more favorable outcome; given the progressive nature of atherosclerosis, further efforts at minimizing modifiable risk factors in young patients at risk for and after acute MI, especially males, are needed. The possible role of the other factors including socioeconomic and psychological status may be considered in further investigations in relation with the acute coronary syndromes.

\section{Riassunto}

Vengono descritte le caratteristiche cliniche, $i$ trattamenti e la prognosi ospedaliera di una popolazione di 2028 pazienti con STEMI, tratti dal Registro Cardiovascolare del Tehran Heart Center, confrontando un gruppo di 109 pazienti di età inferiore a 40 anni con un gruppo di 1919 pazienti di età superiore a 40 anni.

Il profilo di rischio, le caratteristiche clinico-angiografiche ed $i$ trattamenti differivano sostanzialmente nei due gruppi. In particolare il gruppo più giovane presentava una migliore prognosi ospedaliera rispetto ai pazienti più anziani con STEMI.

\section{References}

1. Ounpuu S, Negassa A, Yusuf S. Inter-heart: a global study of risk factors for acute myocardial infarction. Am Heart J 2001; 141: 711-21.

2. Strong K, Wald N, Miller A, Alwan A; WHO Consultation Group.Current concepts in screening for noncommunicable disease: World Health Organization Consultation Group Report on methodology of noncommunicable disease screening. J Med Screen 2005; 12(1): 12-19.

3. Ghadimi H, Bishehsari F, Allameh F, et al. Clinical characteristics, hospital morbidity and mortality, and up to 1year follow-up events of acute myocardial infarction patients: the first report from Iran. Coron Artery Dis 2006; 17(7): 585-91.

4. Sarraf-Zadegan N, Sayed-Tabatabaei FA, Bashardoost N, et al. The prevalence of coronary artery disease in an urban population in Isfahan, Iran. Acta Cardiol 1999; 54(5): 257-63. 
5. Kuulasmaa K, Tunstall-Pedoe H, Dobson A, et al. Estimation of contribution of changes in classic risk factors to trends in coronary-event rates across the WHO MONICA project populations. Lancet 2000; 355: 675-87.

6. Zimmerman FH, Cameron A, Fisher LD, Ng G. Myocardial infarction in young adults: angiographic characterisation, risk factors and prognosis (Coronary Artery Surgery Study Registry). J Am Coll Cardiol 1995; 26: 654-61.

7. Choudhury L, Marsh JD. Myocardial infarction in young patients. Am J Med 1999; 107: 254-61.

8. Sadeghian S, Darvish S, Davoodi G, et al. The association of opium with coronary artery disease. Eur J Cardiovasc Prev Rehabil 2007; 14(5): 715-7.

9. Caro JJ, Migliaccio-Walle K, for the CAPRA (CAPRIE Acutal Practice Rates Analysis) Study Group. Generalizing the results of clinical trials to actual practice: the example of clopidogrel therapy for the prevention of vascular events. Am J Med 1999; 107: 568-72.

10. Yusuf S, Flather M, Pogue J, et al, for the OASIS (Organization to Assess Strategies for Ischaemic Syndromes) Registry Investigators. Variations between countries in invasive and cardiac procedures and outcomes in patients with suspected unstable angina or myocardial infarction without initial ST segment elevation. Lancet 1998; 352: 507-14.

11. Tunstall-Pedoe H, Kuulasmaa K, Amouyel P, Arveiler D, Rajakangas AM, Pajak A. Myocardial infarction and coronary deaths in the World Health Organization MONICA Project. Registration procedures, event rates and case-fatality rates in 38 populations from 21 countries in four continents. Circulation 1994; 90: 583-612.

12. Glover MU, Kuher MT, Warren SE, Vieweg WVR. Myocardial infarction before age 36: risk factor and arteriographic analysis. Am J Cardiol 1982; 49: 160(I-3).

13. Wolfe MW, Vacek JL. Myocardial infarction in the young. Angiographic features and risk factor analysis of patients with myocardial infarction at or before the age of 35 years. Chest 1988; 94: 926-30.

14. Cole JH, Miller JI III, Sperling LS, Weintraub WS. Longterm follow-up of coronary artery disease presenting in young adults. J Am Coll Cardiol 2003; 41: 521-28.

15. Mahonen MS, Mcelduff P, Dobson AJ, Kuulasmaa KA, Evans AE. Current smoking and the risk of non-fatal myocardial infarction in the WHO MONICA Project populations. Tob Control 2004; 13: 244-50.

16. Panagiotakos DB, Rallidis LS, Pitsavos C, Stefanadis C, Kremastinos D. Cigarette smoking and myocardial infarction in young men and women: a case-control study. Int J Cardiol 2007; 116 (3): 371-5.

17. Von Eyben FE, Von Eyben R. Smoking and other major coronary risk factors and acute myocardial infarction before 41 years of age. Scand Cardiovasc J 2001; 35: 25-9.

18. Negri E, La Vecchia C, Nobili A, D'avanzo B, Bechi S. Cigarette smoking and acute myocardial infarction. A case-control study from the GISSI-2 trial. Eur J Epidemiol 1994; 10: 361-6.

19. G Lamm, The epidemiology of acute myocardial infarction in young age groups. In: H Roskamm, Editor, Myocardial infarction at young age, Springer-Verlag, Berkeley (CA) (1981), pp. 5-12.

20. Dolder MA, Oliver MF. Myocardial infarction in young men. Br Heart J 1975; 37: 493-503.

21. Holt BD, Gilpin EA, Henning H, et al. Myocardial infarction in young patients: an analysis by age subsets. Circulation 1986; 74: 712-21.

22. Uhl GS, Farrell PW. Myocardial infarction in young adults: risk factors and natural history. Am Heart J 1983; 105: 548-553.
23. Sozzi FB, Danzi GB, Foco L, et al. Myocardial infarction in the young: a sex-based comparison. Coron Artery Dis 2007; 18(6): 429-31.

24. Andresdottir MB, Sigurdsson G, Sigvaldason H, Gudnason V; Reykjavik Cohort Study. Fifteen percent of myocardial infarctions and coronary revascularizations explained by family history unrelated to conventional risk factors. The Reykjavik Cohort Study. Eur Heart J 2002; 23: 1655-63.

25. Sesso HD, Lee IM, Gaziano JM, Rexrode KM, Glynn RJ, Buring JE. Maternal and paternal history of myocardial infarction and risk of cardiovascular disease in men and women. Circulation 2001; 104: 393-8.

26. Philips B, de Lemos JA, Patel MJ, McGuire DK, Khera A. Relation of family history of myocardial infarction and the presence of coronary arterial calcium in various age and risk-factor groups. Am J Cardiol 2007; 99: 825-9.

27. Garoufalis S, Kouvaras G, Vitsias G, et al. Comparison of angiographic findings, risk factors, and long term followup between young and old patients with a history of myocardial infarction. Int J Cardiol 1998; 67(1): 75-80.

28. Berglud U, Wallentin L, Vonschenk H. Platelet function and plasma fibrinogen and their relations to gender, smoking, habits, obesity and beta-blocker treatment in young survivors of myocardial infarction. Thromb Haemost 1988; 60: 21-4.

29. Kannel WB. Update in the role of cigarette smoking in coronary artery disease. Am Heart J 1981; 101: 319-28.

30. Sattar N, Gaw A. Metabolic syndrome with and without C-reactive protein as a predictor of coronary heart disease and diabetes in the West of Scotland Coronary Prevention Study. Circulation 2003; 108: 414-19.

31. Prescott E, Hippe M, Schnohr P, Hein HO, Vestbo J. Smoking and risk of myocardial infarction in women and men: longitudinal population study. BMJ 1998; 316: 1043-7.

32. Füllhaas JU, Rickenbacher P, Pfisterer M, Ritz R. Longterm prognosis of young patients after myocardial infarction in the thrombolytic era. Clin Cardiol 1997; 20: 993-8.

33. Doughty M, Mehta R, Bruckman D, et al. Acute myocardial infarction in the young-The University of Michigan Experience. Am Heart J 2002; 143: 56-62.

34. Genest JJ, McNamara JR, Salem DN, Schaefer EJ. Prevalence of risk factors in men with premature coronary artery disease. Am J Cardiol 1991; 67: 1185-9.

35. Steg PG, Goldberg RJ, Gore JM, et al; GRACE Investigators. Baseline characteristics, management practices and in-hospital outcomes of patients hospitalized with acute coronary syndromes in the Global Registry of Acute Coronary Events (GRACE). Am J Cardiol 2002; 90: 358-63.

36. Gheorghiade M, Ruzumna P, Borzak S, Havstad S, Ali A, Goldstein S. Decline in the rate of hospital mortality from acute myocardial infarction: impact of changing management strategies. Am Heart J 1996; 131: 250-6.

37. Poloński L, Gasior M, Gierlotka M, et al. Polish Registry of Acute Coronary Syndromes (PL-ACS). Characteristics, treatments and outcomes of patients with acute coronary syndromes in Poland. Kardiol Pol 2007 Aug; 65(8): 861-72.

38. Morillas P, Bertomeu V, Pabón P, et al; PRIAMHO II Investigators. Characteristics and outcome of acute myocardial infarction in young patients. The PRIAMHO II study. Cardiology 2007; 107(4): 217-25.

39. Anderson RE, Pfeffer MA, Thune JJ, et al. High-risk myocardial infarction in the young: the VALsartan In Acute myocardial iNfarcTion (VALIANT) trial. Am Heart $J$ 2008; 155(4): 706-11. 